\title{
Soft and flexible conductive PDMS/MWCNT composites
}

\author{
Hassouneh, Suzan Sager; Yu, Liyun; Skov, Anne Ladegaard; Daugaard, Anders Egede
}

Published in:

Journal of Applied Polymer Science

Link to article, DOI:

10.1002/app.44767

Publication date:

2017

Document Version

Peer reviewed version

Link back to DTU Orbit

Citation (APA):

Hassouneh, S. S., Yu, L., Skov, A. L., \& Daugaard, A. E. (2017). Soft and flexible conductive PDMS/MWCNT composites. Journal of Applied Polymer Science, 134(18), [44767]. https://doi.org/10.1002/app.44767

\section{General rights}

Copyright and moral rights for the publications made accessible in the public portal are retained by the authors and/or other copyright owners and it is a condition of accessing publications that users recognise and abide by the legal requirements associated with these rights.

- Users may download and print one copy of any publication from the public portal for the purpose of private study or research.

- You may not further distribute the material or use it for any profit-making activity or commercial gain

- You may freely distribute the URL identifying the publication in the public portal

If you believe that this document breaches copyright please contact us providing details, and we will remove access to the work immediately and investigate your claim 


\section{Soft and flexible conductive PDMS/MWCNT composites}

2 Suzan S. Hassouneh, Liyun Yu, Anne L. Skov, Anders E. Daugaard*

3 Danish Polymer Centre, Department of Chemical and Biochemical Engineering, Technical

4 University of Denmark, DTU, Søltofts Plads, Building 229, 2800, Kgs. Lyngby (Denmark),

5 adt@kt.dtu.dk

6 Correspondence to: Anders E. Daugaard (adt@kt.dtu.dk).

\section{Abstract}

Conductive elastomers based on MWCNT in polydimethylsiloxane (PDMS) have been prepared by a range of dispersion methods such as ultrasonication, speedmixing and roll milling in combination with physical or covalent modification. The ionic liquid (IL), 1-ethyl-3-methylimidazolium bis(trifluoromethanesulfonyl)imide, was used to pre-disperse MWCNT in a MWCNT/IL-gel that was used for preparation of MWCVNT/PDMS composites. The method was seen to be effective at low levels of MWCNT, but required combination with a roll mill to obtain a stable dispersion at 4 wt\% MWCNT. With higher amounts of MWCNT a reduction in conductivity was observed, which was attributed to a change in morphology occurring between 4 and 5wt\% MWCNT. As an alternative to IL dispersing aids a novel functionalized MWCNT was prepared by free radical polymerization using $\alpha$-methacryloxypropyl-polydimethylsiloxane, which could be used directly for preparation of MWCNT/PDMS composites. Composites prepared by use of the IL dispersion method, use of a roll mill or by use of the f-MWCNT all had conductivities around 0.005-0.01 S/cm and retained conductivity upon extension. 


\section{Introduction}

Conductive elastomers have been a topic of general research interest for a long time and are also commercially available. They are most commonly elastomers filled with conductive particles such as silver, copper, aluminium, nickel, carbon black particles or combinations thereof. Common applications are e.g. electromagnetic screening materials, as flexible electrodes in sensors or in flexible transducers. In particular applications of conductive silicones (polydimethylsiloxane, PDMS) as compliant electrodes for dielectric elastomer (DE) transducers as well as stretchable electronics ${ }^{1}$ have received an increased attention recently ${ }^{2}$. The conductive elastomer must be able to sustain large deformations while remaining conductive as well as remain mechanically stable for millions of cycles. Current commercial solutions cannot be used as a starting point, since these are generally filled to such an extent that adding additional fillers to increase conductivity further is not practically possible.

Several methods have been used to prepare conductive elastomers, such as grafting conductive polymers to soft block copolymers ${ }^{3}$, blending conductive polymers with elastomers ${ }^{4}$ or fabricating stretchable conductors from bacterial cellulose ${ }^{5}$. The preparation of these conductive elastomers is either tedious and/or requires considerable amounts of solvents, which is not preferable from an industrial point of view.

Specifically for PDMS the classical approach of adding conductive particles above the percolation threshold has been investigated in several cases. Various fillers have been used in this respect, such as silver nanowires (AgNWs) ${ }^{6,7}$, exfoliated graphite $\left(\mathrm{EG}^{8}\right)$ and carbon nanotubes (CNTs).

AgNWs were synthesised through the reduction of silver nitrate in the presence of polyvinylpyrrolidone in ethylene glycol ${ }^{9,10}$, resulting in a suspension that was used for elastomer preparation. The prepared elastomers containing the AgNWs was shown to have a high conductivity, though the conductivity was shown to decrease when the elastomer was strained ${ }^{6,7}$. 
Similarly, CNTs having single or multiple graphene layers rolled into cylinders as single walled carbon nanotubes (SWCNT) or multi walled carbon nanotubes (MWCNT) $)^{11-13}$ have received much attention because of their excellent mechanical, thermal and electrical properties ${ }^{14,15}$. CNTs are generally interesting as nanofillers for conductive elastomers, since their percolation thresholds are generally very low and conductive networks are achieved at concentrations below $1 \mathrm{wt} \%$, due to their high aspect ratio ${ }^{12,15}$. However, a drawback of CNTs is their poor dispersion in elastomers, due to the formation of agglomerates as a result of the strong van der Waals force of interaction between the $\mathrm{CNTs}^{16,17}$. In order to achieve a better dispersion of CNTs in elastomers, covalent and non-covalent modifications can be used. Covalent modification includes, amongst others, grafting functionality to the CNT surface ${ }^{18-20}$, while non-covalent modification usually involves mixing with a low molecular weight compound such as e.g. ionic liquids (ILs) or other compatibilizers. A broad range of ILs are currently available as either organic-inorganic salts or as purely organic salts and can be tailored to specific properties based on the choice of cation and anion such as 1-allyl-3-methyl imidazolium, 1ethyl-3-methylimidazolium or tetralkyl ammonium in combination with bis(trifluoromethylsulphonyl)imide, chloride or e.g. tetrafluoroborate. The use of ILs has generally been observed to improve the dispersion of CNT in polymers, where in particular the imidazolium based ILs have resulted in composites with an increased conductivity ${ }^{13,21-29}$. For PDMS, Oh et al. ${ }^{22}$ recently demonstrated how the use of an IL as a dispersing agent resulted in well dispersed SWCNT/PDMS composites. Their focus was on development of soft systems with a low amount of SWCNT (1.6 wt\%) and they used large amounts of IL in order to use it both as a dispersing agent as well as a plasticizer to obtain softer materials. They did not report the conductivities obtained by their process, but their composites were clearly above the percolation threshold. 
In addition to effective dispersion aids, effective mixing methods are required in order to disperse carbon materials in PDMS. Previous investigations in our group showed the effect of different methods of dispersion such as mechanical mixing, ultrasonication and high speed mixing in a Speedmixer and how that influenced the quality of an expanded graphite/PDMS composite ${ }^{30}$. From the available techniques in particular speedmixing and the use of a roll mill would be suitable methods for use in industrial production to prepare high quality composites.

The purpose of this study was to identify an industrially viable process for preparation of a soft flexible conductive PDMS composite with a lower degree of filling compared to commercially available materials. Therefore several different approaches using different dispersing aids or mixing methods were tested on an industrial grade of MWCNT.

\section{Experimental}

\section{Materials and methods}

MWCNTs (NC7000) were purchased from Nanocyl S.A. (Belgium). The MWCNTs is reported by the supplier to have an average diameter of $9.5 \mathrm{~nm}$, an average length of $1.5 \mu \mathrm{m}$ and a surface area of 250-300 $\mathrm{m}^{2} / \mathrm{g}$. The ionic liquid, 1-ethyl-3-methylimidazolium bis(trifluoromethanesulfonyl)imide and heptane were acquired from Sigma Aldrich. Elastosil RT625 and the inhibitor PT-88 were purchased from Wacker Chemie AG (Germany). Elastosil RT625 is a two-component system, in which component A consists of vinyl-terminated PDMS and a crosslinker, and component B consists of vinyl-terminated PDMS and a catalyst, among other components such as fillers and additives. The two components are mixed in a 9:1 ratio (A:B). In order to prepare the samples the zero volt ionizer from Desco Industries Inc., an ultrasonic device UP200S from Heilscher, a mechanical mixer Eurostar from IKA Labortechnik, a rotary-evaporator Laborota 4003 from Heidolph, a Speedmixer ${ }^{\mathrm{TM}}$ from Flack Tek Inc. and a three-roll mill from EXAKT Advanced Technologies GmbH have been 
used. Thermo gravimetric analysis (TGA) was performed in a nitrogen atmosphere on a Q500 from TA instuments with a heating rate of $10^{\circ} \mathrm{C} / \mathrm{min}$ from RT to $800^{\circ} \mathrm{C}$. Fourier Transform Infrared spectroscopy (FT-IR) was performed on a Thermo-Fischer is50 FT-IR with a universal attenuated total reflection (ATR) sampling accessory on a diamond crystal. The rheological measurements were carried out on a controlled stress rheometer (AR2000) from TA Instruments at $20^{\circ} \mathrm{C}$ using samples with a diameter of $25 \mathrm{~mm}$ and a thickness of $1 \mathrm{~mm}$.

\section{Procedures}

\section{General procedure for preparation of MWCNT/PDMS composites}

For samples with IL as dispersion aid, MWCNTs and the IL, 1-ethyl-3-methylimidazolium bis(trifluoromethanesulfonyl)imide, in the desired ratio were weighed in a mortar and ground with a pestle until gelation was observed. Either pristine MWCNTs or the MWCNT/IL-gel was subsequently dispersed in heptane at $1 \mathrm{mg}$ MWCNT/mL and ultrasonicated for 14 minutes in 2 minute intervals. After ultrasonication, component A of Elastosil RT625 was added and the mixture was stirred for 6 hours at $900 \mathrm{rpm}$. The heptane was evaporated from the mixture using a rotary-evaporator. Thereafter component B of Elastosil RT625 was added and the mixture was speed-mixed for 5 minutes at $2750 \mathrm{rpm}$. The composite was cast onto a glass plate with a $1 \mathrm{~mm}$ thick frame, where after another glass plate was placed on top of the sample, thus sandwiching the sample. The glass plates were clamped together and the sample was cured in the oven for 10 minutes at $115^{\circ} \mathrm{C}$, resulting in a fully cured PDMS/MWCNT/IL composite.

\section{Roll mill dispersion - General procedure for preparation of MWCNT/PDMS composites}

MWCNTs or MWCNT/IL gels, as well as both Elastosil RT625 components together with 1 wt\% inhibitor with respect to the PDMS (to delay the curing process), were weighed into the speed mixing cup. The mixture was then speed-mixed for 5 minutes at $2750 \mathrm{rpm}$ and was ready to be dispersed in the roll mill. The gap between the first and second rolls was set to $30 \mu \mathrm{m}$, while the gap between the 
second and third rolls was set to $15 \mu \mathrm{m}$ and the speed of the third roll was set to $100 \mathrm{rpm}$. The sample was roll-milled six times until the mixture was uniformly blended and finally coated and cured thermally for 10 minutes at $115^{\circ} \mathrm{C}$.

\section{Poly(MPDMS) grafted MWCNT by free radical polymerization}

The MWCNTs (1.5 g) were dispersed in a mixture of anisole $(27 \mathrm{~mL})$ and toluene $(220 \mathrm{~mL})$ by ultrasonication for $10 \mathrm{~min}$. The monomer $\alpha$-methacryloxypropyl-polydimethylsiloxane (MPDMS, $\mathrm{M}_{\mathrm{n}}=700 \mathrm{~g} / \mathrm{mol}, 33 \mathrm{~mL}, 31.8 \mathrm{~g}$ ) and azobisisobutyronitrile (AIBN, $0.045 \mathrm{~g}, 2.4 \mathrm{mmol}$ ) were added to the dispersed MWCNT and the reaction mixture was bubbled with nitrogen for 20 min and thereafter heated to $65^{\circ} \mathrm{C}$. The mixture was heated for $18 \mathrm{~h}$ under nitrogen and the product was isolated by precipitation into $\mathrm{MeOH}$ (3 L), followed by filtration using a $0.4 \mu \mathrm{m}$ PTFE filter, resulting in a black viscous solid in a quantitative yield. The raw product could be used directly for preparation of composites or rinsed for free polymer by washing with toluene and drying in vacuo. The raw product was speed-mixed with both components of the PDMS (A and B) at $2750 \mathrm{rpm}$ for 5 minutes. The sample was coated on a glass plate and cured in an oven for 10 minutes at $115^{\circ} \mathrm{C}$.

IR (cm ${ }^{-1}$ ): 3100-2800 (CH stretch); 1728 (CO stretch); 1255 (CO stretch); 1100 and 1015 (Si-O stretch) and 787 (Si-C stretch).

\section{Results and discussion}

The process developed by Oh et al. ${ }^{22}$ for SWCNT was adopted to higher amounts of an industrial grade MWCNT to investigate it as a pathway towards highly conductive soft elastomers. The preparation process is outlined in Figure 1. 


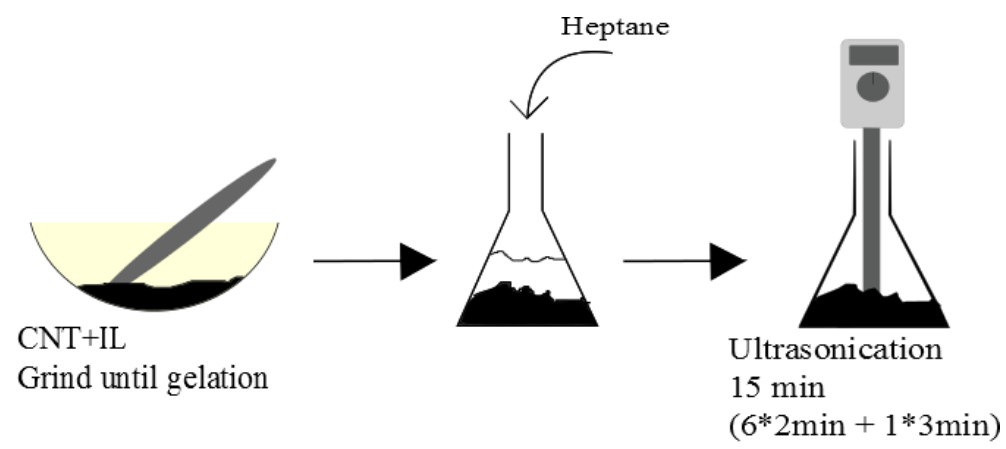

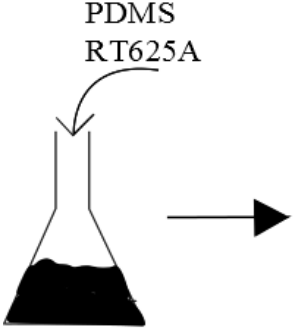

PDMS

RT625B

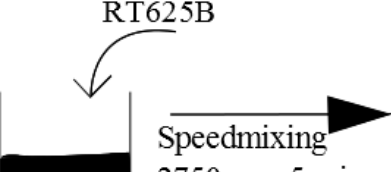

$2750 \mathrm{rpm} 5 \mathrm{~min}$
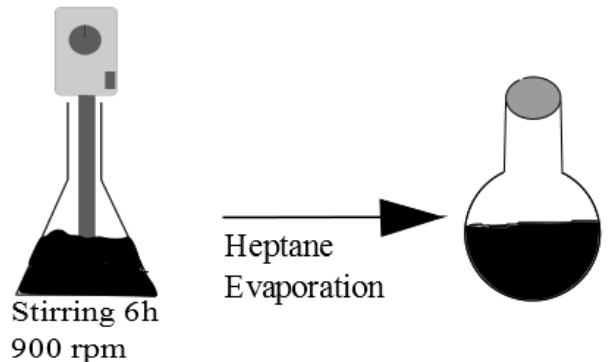

Evaporation

$900 \mathrm{rpm}$

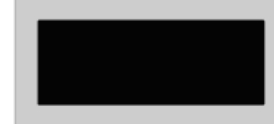

Figure 1: Schematic overview of the dispersion procedure using IL to disperse MWCNT in PDMS followed by coating and thermal curing of the MWCNT/PDMS composites. soluble in heptane this allowed direct mixing of the liquid PDMS pre-polymer into the MWCNT dispersion. This was additionally sonicated, stirred and speedmixed to provide a fully dispersed

PDMS mixture that could ultimately be cured by thermal curing. The process applies a combination of dispersion methods in the form of the IL-gel, ultrasonication and speedmixing to obtain a stable dispersion of the MWCNT in PDMS.

The conductive properties of the prepared elastomers are directly linked to the quality of the dispersion and dielectric spectroscopy (DRS) was therefore used to determine to assess the quality of the dispersion as shown in Figure 2-left. 

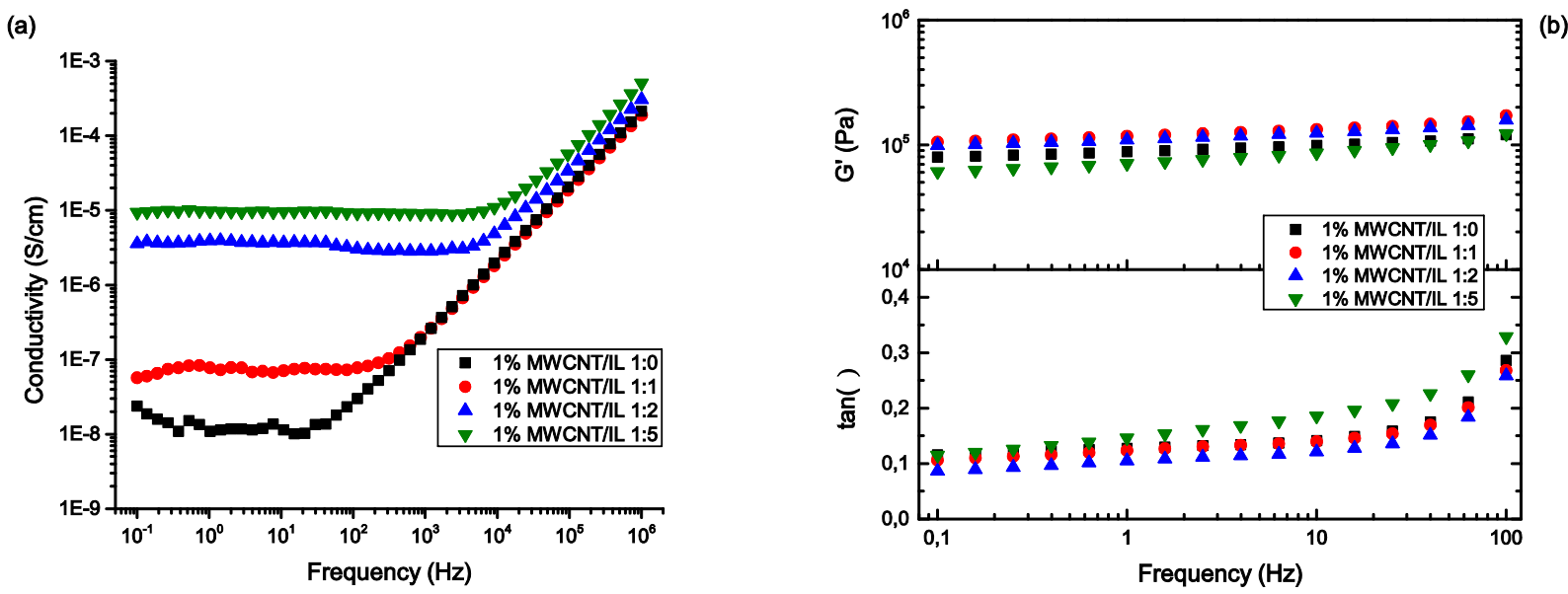

150

151

152

Figure 2: 1 wt\% MWCNT/PDMS composites with a MWCNT/IL ratio from 1:0 to 1:5. a) Conductivities at 20다 ${ }^{\circ}$; b) Linear viscoelastic properties at $20^{\circ} \mathrm{C}$.

As shown in Figure 2a the PDMS/MWCNT composites all have a direct current plateau at lower frequencies, which is indicative for conductive materials. The level of the plateau, corresponding to the conductivity, increased with increasing amounts of IL. This is a result of an improved dispersion of the MWCNT in the matrix, since the IL is well incorporated (samples are unchanged after a year) and does not lead to a higher conductivity of the matrix, as can be seen from a reference experiment using only IL and the Elastosil 625 (SI-Figure 1). One of the disadvantages of using ILs as dispersing aids would be the cost of material as well as the impact on network integrity, when the loading becomes significantly higher than the MWCNT. It is well known that foreign compounds will leach out of the PDMS over time and the stability of the matrix was therefore investigated by rheology as shown in Figure 2b. Here it can be seen that increasing the amounts of IL relative to the MWCNT results in similar rheological behaviour, though the highest ratio tested (MWCNT/IL 1:5) shows a minor increase in $\tan (\delta)$ at higher frequencies. Generally, all of the composites show a good mechanical integrity and an effective dispersion, though increasing the MWCNT/IL ratio to 1:5 can be seen to be of negligible effect with respect to conductivity. 

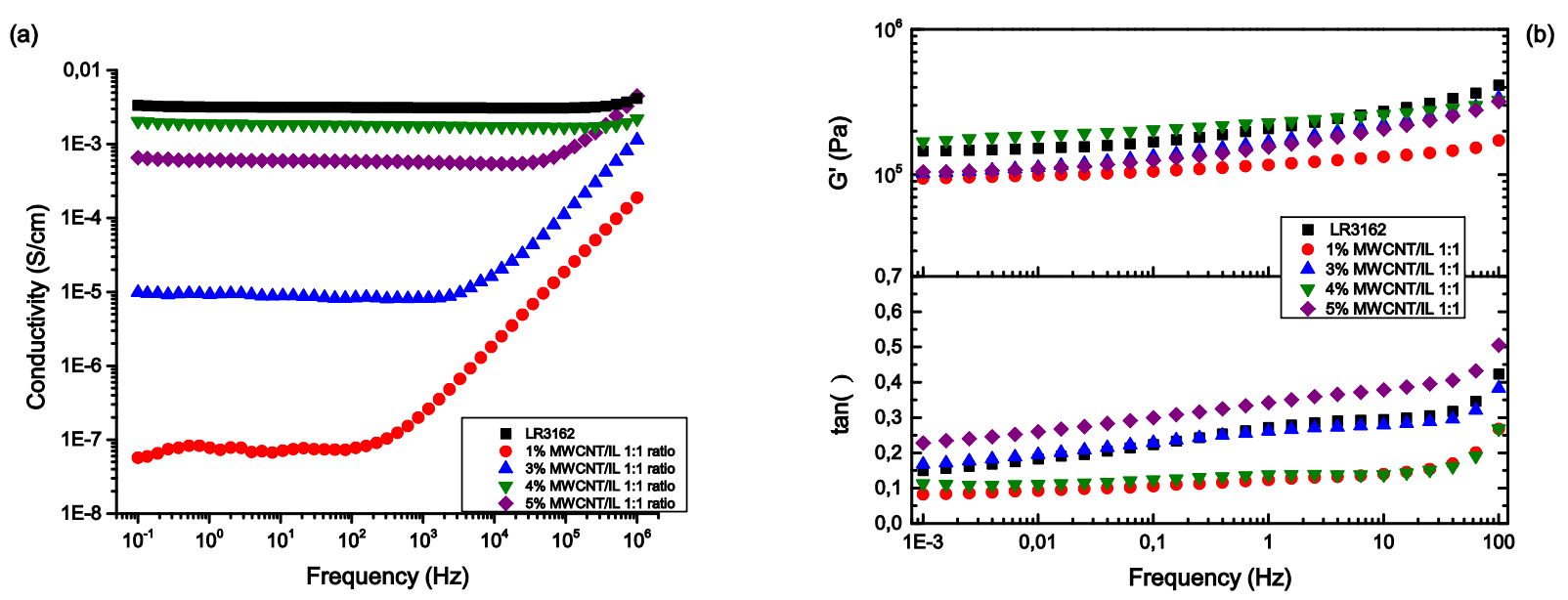

Figure 3: MWCNT/IL PDMS composites with 1-5 wt\% MWCNT at a MWCNT/IL ratio of 1:1. a) Conductivities at $20^{\circ} \mathrm{C}$; b) Linear viscoelastic properties at $20^{\circ} \mathrm{C}$.

Increasing the content of MWCNT in the composites resulted in an effective increase in conductivity up to a loading of 4 wt $\%$ MWCNT (Figure 3a). At this point an upper limit of effective incorporation was reached as is often seen for nanocomposites, where increasing the loading further resulted in a decreased conductivity. This illustrates the necessary balance between loading, effectiveness of the filler, as well as the degree of dispersion that is critical for an effective nanocomposite. The mechanical stability of the materials was also investigated for higher amounts of MWCNT as shown in Figure 3b. With increased content of MWCNT and IL in the network, viscous losses increase for some of the systems, though this phenomenon is not reproducible and is attributed to small agglomerates resulting from minor sample to sample variations.

Based on the results above it can be seen that the MWCNT/PDMS composites approach the conductivity of the commercially available LR3162, which contains $40 \mathrm{wt} \%$ carbon black. In an effort to increase the dispersion and thereby the conductivity even further composites using a mixing ratio of MWCNT/IL 1:2 were also investigated as shown in Figure 4 


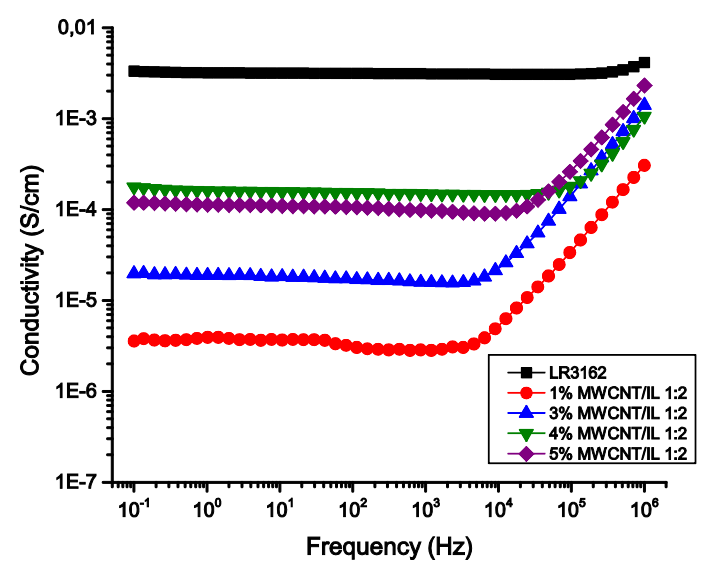

Figure 4: Conductivities of MWCNT/IL PDMS composites with a MWCNT content from 1-5 wt\% with a MWCNT/IL ratio of $1: 2$ at $20^{\circ} \mathrm{C}$.

Here it is clear that the system is not sufficiently robust at high levels of MWCNT. The conductivity increases for the 1 and 3 wt\% MWCNT composites with a mixing ratio of 1:2 compared to the 1:1 mixing ratio shown above. However, increasing either the amount of MWCNT to 4 or 5 wt $\%$ or the amount of IL (compared to 1:1) does not result in an effective dispersion of the MWCNT. The upper limit observed in conductivity for the composites with 4-5 wt\% MWCT and varying amounts of IL indicates that there is a change in dispersion occurring at this specific loading. To investigate this change, a range of composites with varying IL content and high amounts of MWCNT (4-5wt\%) was investigated by scanning electron microscopy (SEM) as shown in Figure 5. 


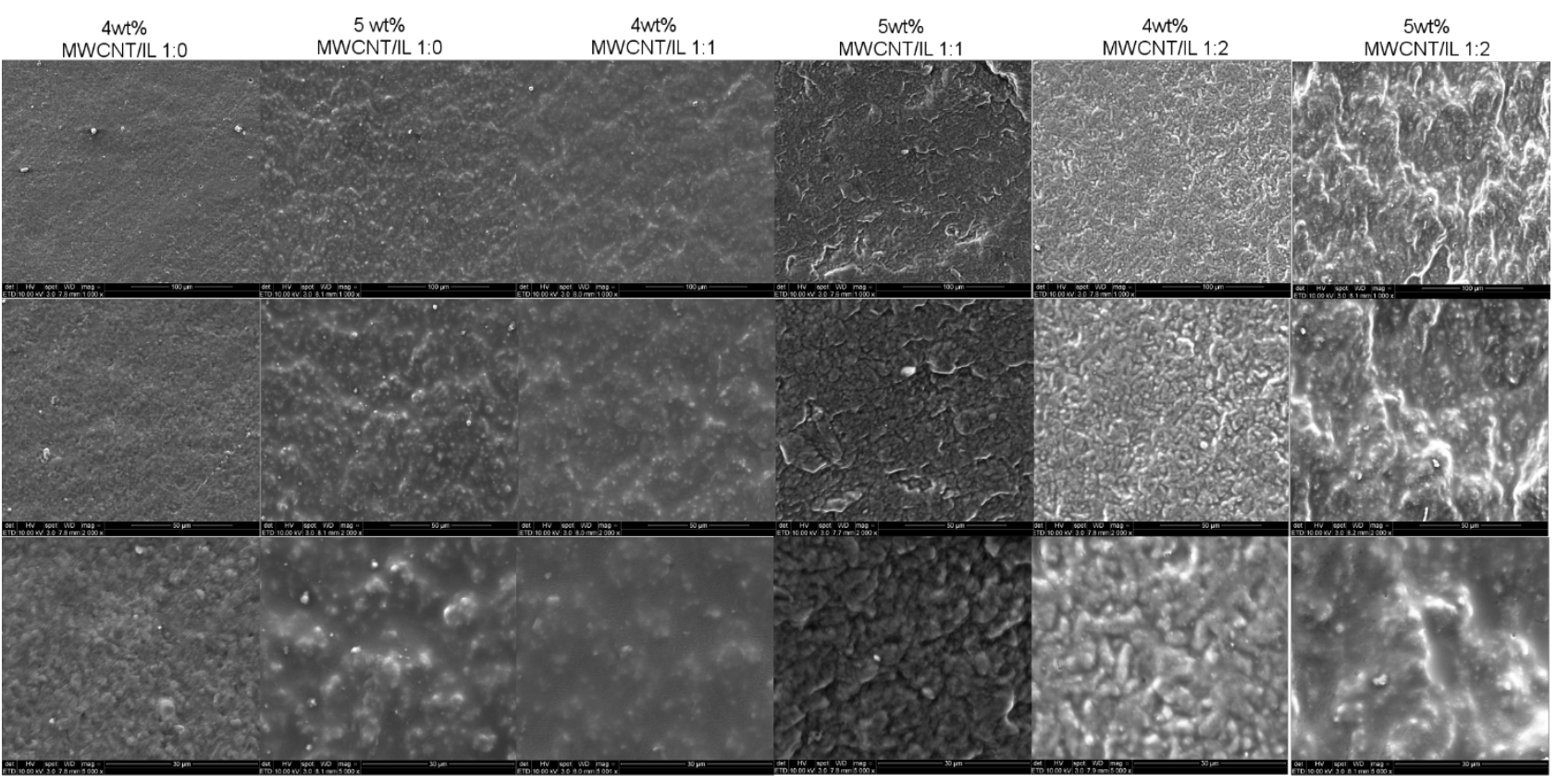

Figure 5: SEMs of the composites containing 4-5wt\% MWCNT and 0-2 wt\% IL. The particles (white spots) seen in the SEMs are silica particles from the Elastosil RT625.

208 From the micrographs it is clear that samples without IL and 4-5 wt\% MWCNT have a homogeneous appearance, where the MWCNT appears well distributed. With addition of a small amount of IL the picture is unchanged for $4 \mathrm{wt} \%$ MWCNT and the elastomer appears very similar. However, the $5 \mathrm{wt} \%$ of MWCNT/IL 1:1 shows a distinctly different morphology. With a higher amount of IL (MWCNT/IL 1:2) this morphology is observed for all the samples. In addition to this the samples begin to accumulate charge, which is normally observed for MWCNT composites

214 below the percolation threshold ${ }^{31}$. It is unclear exactly what is happening at this composition, but it directly correlates to the low conductivities observed for these samples both for the $5 \mathrm{wt} \%$ MWCNT/IL 1:1 and for MWNCT/IL 1:2 elastomers.

These results thereby show that moderate amounts of IL is quite effective as a dispersing aid at low amounts of MWCNT, whereas larger amounts of IL or higher amounts of MWCNT results in in a less efficient dispersion. 
Due to the good results on $4 \mathrm{wt} \%$ MWCNT (MWCNT/IL 1:1) this system was used to investigate the effect of using roll milling as an additional dispersion method. The process outlined in Figure 1 was additionally followed by a final treatment on a roll mill to homogenize the samples. To see the effect of using the roll mill both samples with and without IL were investigated as shown in Figure

6.

Figure 6: Conductivities of 4 and 5 wt\% MWCNT/PDMS composites without and with IL dispersed by speedmixing

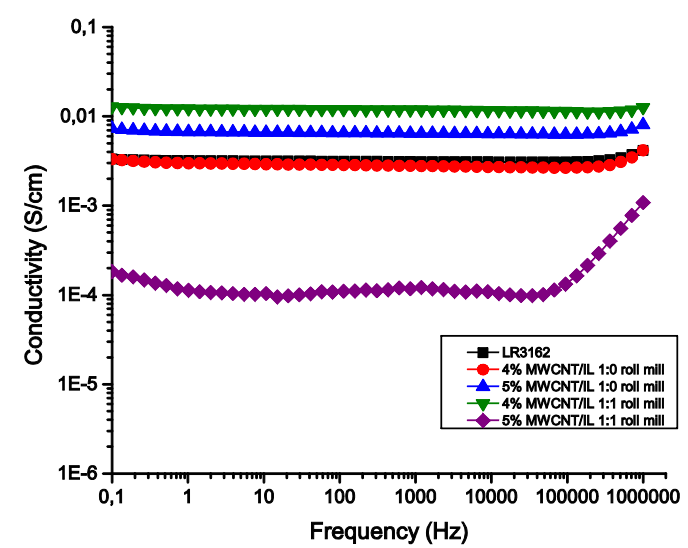
followed by a roll mill treatment measured at $20^{\circ} \mathrm{C}$.

Here it is clear that roll milling in combination with speedmixing provides a very efficient dispersion method. For all the roll milled samples the direct current plateau has a much earlier onset compared to the MWCNT/IL or pristine MWCNT composites. This clearly underlines that the roll mill provides a much more effective dispersion compared to the earlier mentioned methods. In the roll milled MWCNT composites without IL there is not much difference between the 4 and 5 wt\% MWCNT composites. Both samples are well dispersed and provide a high conductivity comparable to that of LR3162. In contrary to this, for the samples that were pre-dispersed as an IL-gel with 4 and 5 wt\% MWCNT a difference of several orders of magnitude in conductivity was seen. The $4 \mathrm{wt} \%$ sample can be seen to give a slightly higher conductivity than LR3162, whereas the $5 \mathrm{wt} \%$ 

elastomer films were investigated by rheology as shown in Figure 7.
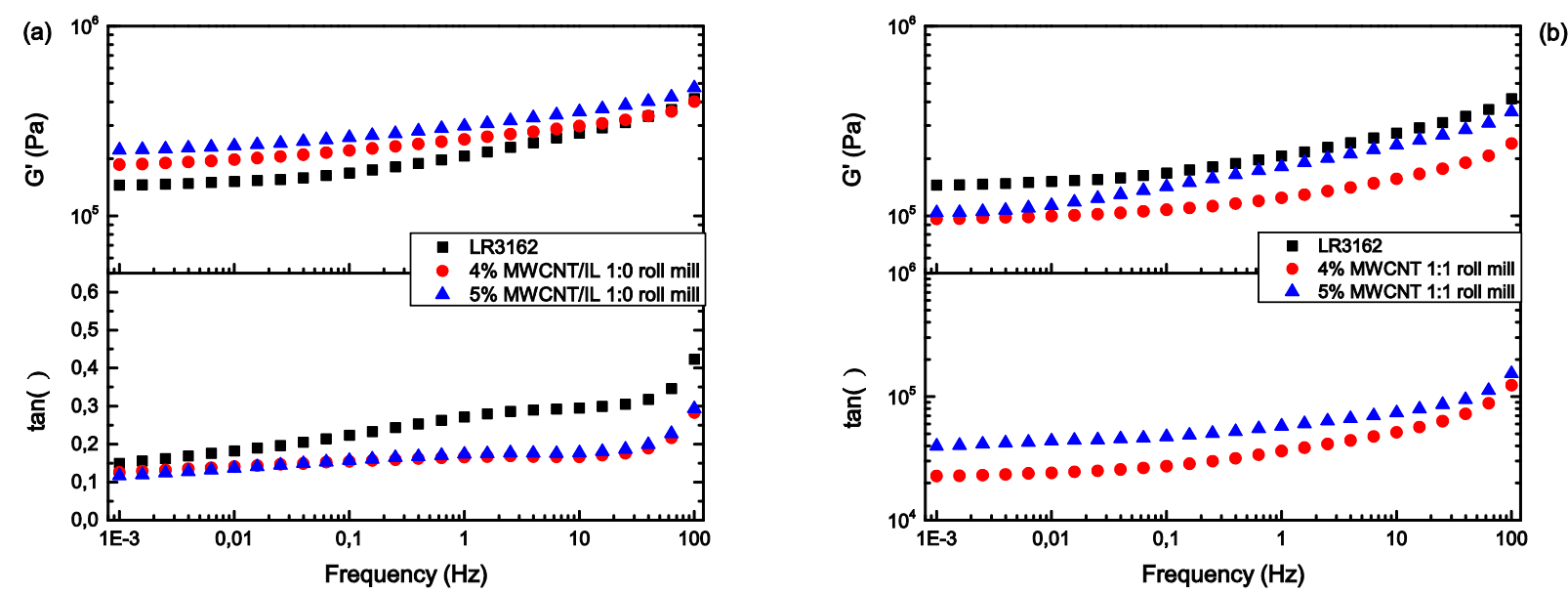

Figure 7: Linear viscoelastic properties of the roll milled 4 and 5 wt\% MWCNT/PDMS composites without (a) and with (b) IL measured at $20^{\circ} \mathrm{C}$.

For the samples without IL (Figure 7a), G’ increases in line with increasing concentration of MWCNTs and the samples are stiffer that the commercially available Elastosil LR3162. However, when the MWCNTs are pre-dispersed using the IL the composites become softer (Figure 7b). The expected influence of MWCNT on a polymer matrix would be an increase in G' as a function of loading, as e.g. observed on thermoplastic systems such as PP/MWCNT composites. ${ }^{31}$ For elastomers there is an additional effect from the formation of the network, where an improved dispersion influences network integrity and thereby affects the mechanical stability, which has to be taken into consideration in the design of the elastomer composition. ${ }^{32}$ For elastomers, the pristine MWCNT affects the network to an insignificant degree and results in only a moderate increase in G'. In contrast to this, the addition of the IL in combination with the improved efficiency of the dispersion by the roll mill, results in a significant influence on the mechanical properties. In particular for the $5 \mathrm{wt} \%$ sample the softening effect of the IL is clear, where a significant increase in 
$\tan (\delta)$ can be observed at low frequencies indicating a much weaker network and significant viscous loss in the network.

259

260

In general, the additional step of using the roll mill resulted in a more robust procedure that provided a very efficient dispersion. However, the positive effects of using the IL in the predispersion step is counterbalanced with a significant influence on the network that is not desirable. In addition to this, the added cost of the procedure and the only moderate increase compared to the pristine MWCNT does not make this procedure competitive.

\section{Functionalized MWCNT as a dispersion method}

In addition to the use of mechanical mixing methods functionalization of nanomaterials are a wellknown pathway towards improved dispersion in nanocomposites. However, as underlined by $\mathrm{Grady}^{33}$, the use of functionalized materials will be a balance between improved dispersion and loss of MWCNT structure due to the functionalization, which ultimately results in a lower ultimate conductivity as well as a lower ultimate tensile strength of the MWCNT. Therefore a simple preparation method affording a low degree of modification as well as a product that could easily be used in a subsequent PDMS network curing reaction was developed as illustrated in Scheme 1.
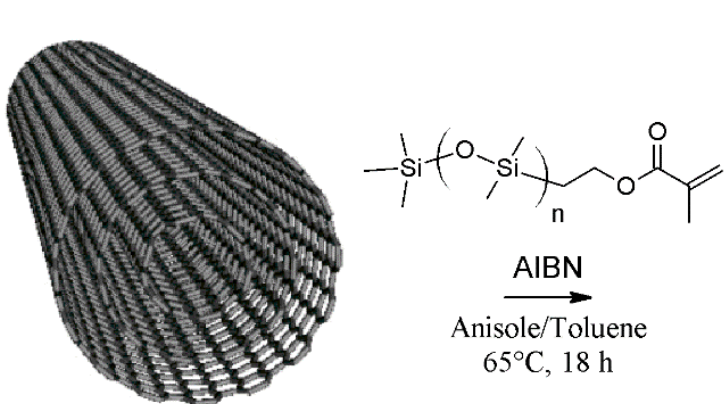
$65^{\circ} \mathrm{C}, 18 \mathrm{~h}$

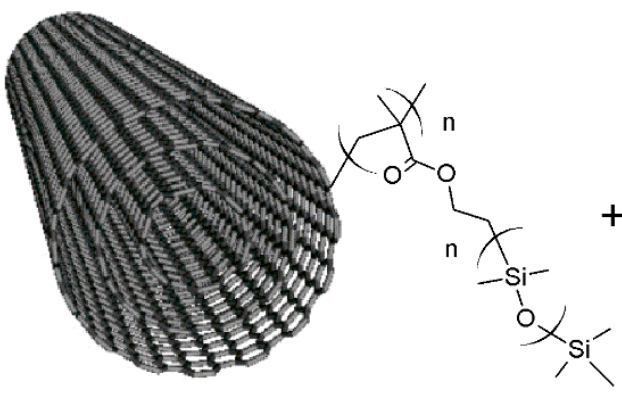

MWCNT- Poly(MPDMS)

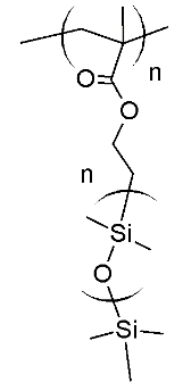

Poly(MPDMS)

Scheme 1: Synthesis of poly(MPDMS) grafted MWCNT (f-MWCNT) by in-situ free radical polymerization of MPDMS resulting in a f-MWCNT in excess free poly(MPDMS). 
A free radical polymerization of MPDMS was conducted in a dispersion of pristine MWCNT in a mixture of toluene and anisole. The method is very simple to conduct and has the advantage that it can directly be up-scaled for large amounts of modified MWCNT. The purification process was a simple precipitation in methanol, which allows both the MWCNT as well as any free polymer formed to be isolated, while excess monomer will be removed. As shown in Figure 8-left the amount of grafted polymer on the MWCNT could be determined from thermogravimetric analysis (TGA).

(a)

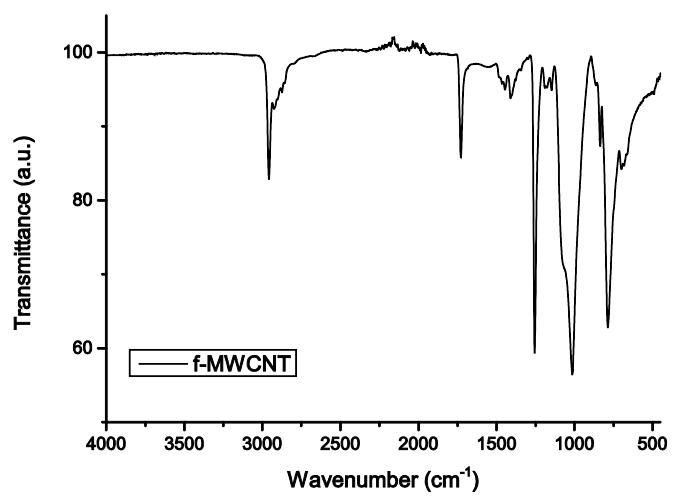

(b)

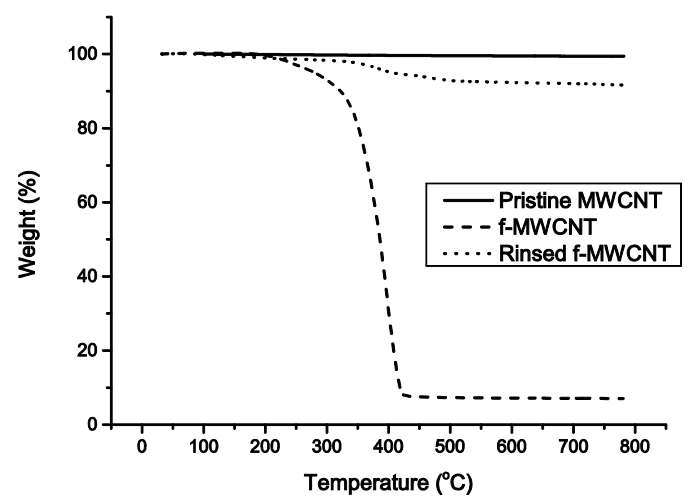

Figure 8: TGA of the pristine MWNCT, rinsed f-MWCNT and the unpurified raw product (f-MWCNT) that was used directly for the composite preparation (a); IR spectrum of the raw product showing the main peaks from PDMS (b).

The free radical process results in a combination of free poly(MPDMS) and surface grafted poly(MPDMS)-MWCNT (f-MWCNT). By rinsing the precipitated raw product with toluene, it was possible to remove the excess free polymer, as seen from the significantly lower weight loss observed in TGA. This rinsed product still shows a weight loss compared to the pristine MWNCT, which proves the polymer is covalently bound to the MWCNT ( $8 \mathrm{wt} \%$ polymer). The rinsed fMWCNT is a hard and brittle material of similar appearance to pristine MWCNT, whereas the raw product is a well dispersed greasy solid. The raw product contained $7 \mathrm{wt} \%$ MWCNT, while the remaining sample mass is either free or covalently bound poly(MPDMS). The polymer structure 
was confirmed by FT-IR (Figure 8b), where the clear methacrylate stretch at $1728 \mathrm{~cm}^{-1}$ in combination with the PDMS stretches at 1100, 1015 and $787 \mathrm{~cm}^{-1}$ and the absence of the methacrylic double bond confirmed the structure of the polymer. The prepared raw product is a homogeneous black, viscous material, which could be used directly after isolation for preparation of PDMS networks (concentration is given as the amount of MWCNT in the elastomer). The functionalized materials required much lower intensity dispersion methods and were just mixed with PDMS prepolymers in a speedmixer and afforded elastomers with conductivities as shown in Figure 9a.

(a)
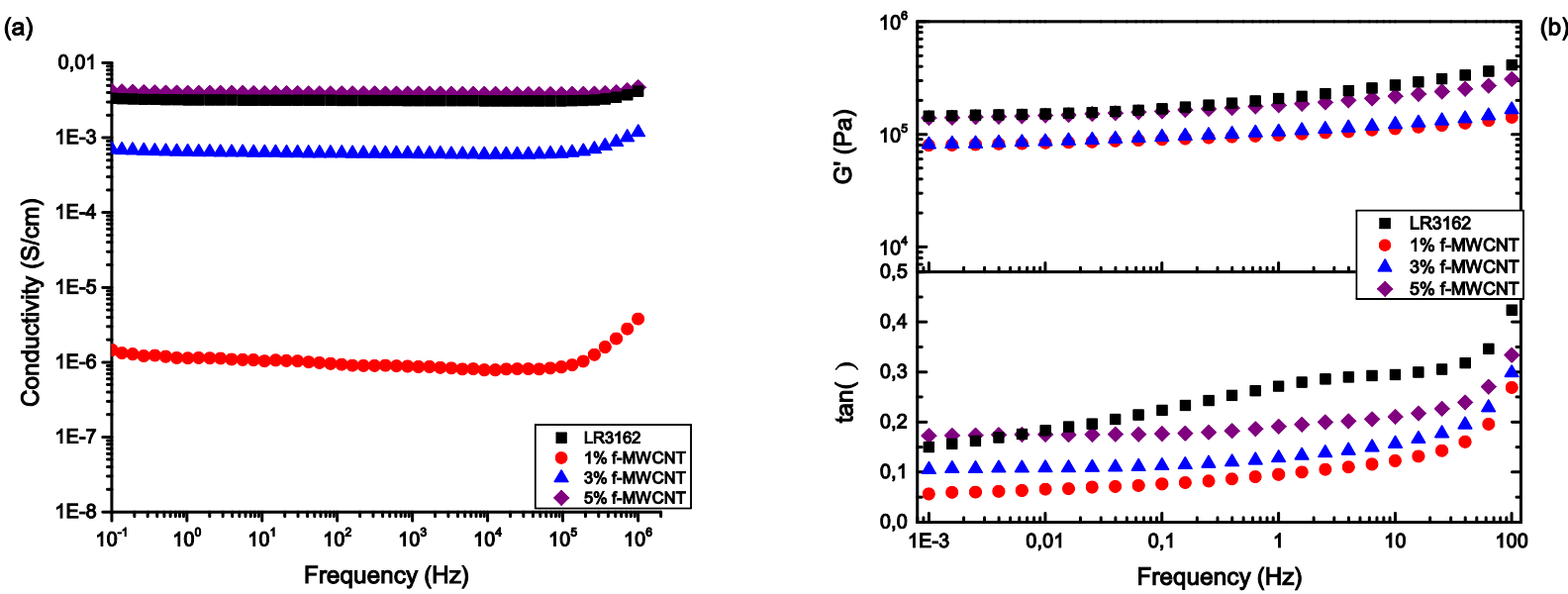

302 303

Figure 9: PDMS composites containing 1-5 wt\% f-MWCNT. a) Conductivities at $20^{\circ} \mathrm{C}$; b) Linear viscoelastic response at $20^{\circ} \mathrm{C}$.

The functionalized material results in a direct current plateau 2 orders of magnitude above that of pristine MWCNT and 1 decade above that from the IL-gel procedure for $1 \mathrm{wt} \%$ MWCNT (Figure 2a). In addition to this, the onset of the plateau is at much higher frequencies for the f-MWCNT composites, compared to the pristine MWCNT and the MWCNT/IL composites, indicating a very efficient dispersion. Increasing the content of the f-MWCNT to 3 and $5 \mathrm{wt} \%$ resulted in conductivities on the order of the commercial LR3162. The materials show elastic properties comparable to that of LR3162 (Figure 9b), with a slightly lower G' for the f-MWCNT at 1 and 
$3 \mathrm{wt} \%$. The losses are significantly lower than for the IL-gel dispersed MWCNT as well as for LR3162. The combination of both a low G' as well as low losses shows that the network integrity has been maintained. The functionalization also had a very positive effect on mixing and handling, where these materials readily disperses in solvent or the PDMS pre-polymer matrix by manual mixing.

Finally, the impact on conductivity by extension of the elastomers for the f-MWCNT and the MWCNT/IL 1:1 roll mill dispersed samples was compared to the commercial LR3162 at different strains as shown in Figure 10.

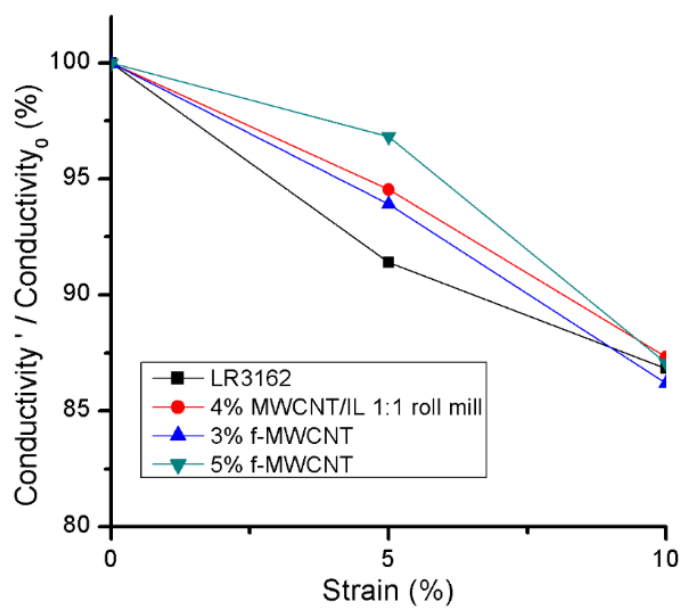

Figure 10: Change in conductivity as a function of strain for the most promising elastomer candidates plotted relative to the respective original conductivities measured at $20^{\circ} \mathrm{C}$.

From the plot of relative conductivity of the elastomers it can be seen that the f-MWCNT as well as the IL dispersed MWCNT retain their initial conductivity to a greater extent than the commercial reference at 5\% strain, whereas all of the samples show an $13 \%$ reduction in conductivity at $10 \%$ strain. The stretchability of particularly the $5 \mathrm{wt} \%$ f-MWCNT shows a promising potential for this type of material at small strains, where there is a significant improvement compared to the reference material. This is particularly important for applications in joints and connections where a constant conductivity of such a flexible material is required. 
The use of IL/MWCNT-gels as a pre-dispersion method was evaluated for the preparation of highly filled MWCNT/PDMS composites. IL-gels were shown to be effective in dispersing the MWCNT, but the method was not sufficiently robust at higher loading of MWCNT. In order for the method to be applicable it is required that a high reproducibility as well as an easy handling can be obtained. The method allowed the preparation of highly conductive MWCNT/PDMS composites with 4 wt\% MWCNT (at a MWCNT/IL 1:1 ratio) but the amount of manual work involved in the preparation was deemed not to give a significant high return in the form of conductivity compared to the other processes. SEM analysis of the elastomers showed that the observed reduction in conductivity at higher amounts of MWCNT could be attributed to a change in morphology occurring between 4 and 5wt\% MWCNT (or at 4wt\% MWCNT with high IL load).

When the IL method was combined with a roll mill the dispersion was more effective and more material. However, under these conditions also pristine MWCNT could be dispersed to a similar degree by combination of a speedmixing step followed by treatment in the roll mill.

As an alternative to the roll mill a novel type of PDMS grafted f-MWCNT was prepared and tested using speedmixing as dispersion method. The f-MWCNTs were easier to handle and facilitated a stable dispersion in PDMS pre polymers and composites with conductivities comparable to LR3162 without the requirement of the roll mill treatment. The f-MWCNT procedure is an additional step, but the approach is a simple and up-saleable process without the requirement of rigorous and time consuming purification steps. 
Finally, the conductivity of the elastomers during extension was confirmed up to $10 \%$ strain for selected samples, showing a promising improvement in retained conductivity at $5 \%$ strain and comparable results at $10 \%$ strain compared to LR3162.

Overall the approaches illustrate that in order to obtain a good dispersion of MWCNT in PDMS and obtain a high conductivity of the elastomer a sequence of both dispersion aids as well as effective mechanical mixing methods are required. Either speedmixing/US in combination with a roll mill or f-MWCNT in combination with speedmixing was identified as the most effective approaches to obtain highly conductive PDMS composites with maintained mechanical integrity.

\section{Acknowledgements}

The authors would like to thank the Danish Agency for Science and Innovation Fund Denmark for financial support.

\section{References}

(1) Wagner, S.; Bauer, S. MRS Bull. 2012, 37, 207-213.

(2) Rosset, S.; Shea, H. R. Appl. Phys. A Mater. Sci. Process. 2013, 110, 281-307.

(3) Stoyanov, H.; Kollosche, M.; Risse, S.; Waché, R.; Kofod, G. Adv. Mater. 2013, 25, 578583.

(4) Hansen, T. S.; West, K.; Hassager, O.; Larsen, N. B. Adv. Funct. Mater. 2007, 17, 30693073.

(5) Liang, H.-W.; Guan, Q.-F.; Zhu, Z.-; Song, L.-T.; Yao, H.-B.; Lei, X.; Yu, S.-H. NPG Asia Mater. 2012, 4, e19.

(6) Akter, T.; Kim, W. S. ACS Appl. Mater. Interfaces 2012, 4, 1-3.

(7) Yun, S.; Niu, X.; Yu, Z.; Hu, W.; Brochu, P.; Pei, Q. Adv. Mater. 2012, 24, 1321-1327.

(8) Hassouneh, S. S.; Daugaard, A. E.; Skov, A. L. Macromol. Mater. Eng. 2015, 300, 542-550.

(9) Korte, K. E.; Skrabalak, S. E.; Xia, Y. J. Mater. Chem. 2008, 18, 437-441.

(10) Sun, Y.; Gates, B.; Mayers, B.; Xia, Y. Nano Lett. 2002, 2, 165-168.

(11) Bokobza, L. Polymer 2007, 48, 4907-4920. 
380

381

382

383

384

385

(12) Subramaniam, K.; Das, A.; Stöckelhuber, K. W.; Heinrich, G. Rubber Chem. Technol. 2013, 86, 367-400.

(13) Peng, R.; Wang, Y.; Tang, W.; Yang, Y.; Xie, X. Polymers 2013, 5, 847-872.

(14) Seo, D. K.; Kang, T. J.; Kim, D. W.; Kim, Y. H. Nanotechnology 2012, 23, 75501.

(15) Moniruzzaman, M.; Winey, K. I. Macromolecules 2006, 39, 5194-5205.

(16) Ajayan, P. M. Nature 2007, 447, 1066-1068.

(17) Fritzsche, J.; Lorenz, H.; Klüppel, M. Macromol. Mater. Eng. 2009, 294, 551-560.

(18) Gojny, F. H.; Schulte, K. Compos. Sci. Technol. 2004, 64, 2303-2308.

(19) Ramanathan, T.; Liu, H.; Brinson, L. C. J. Polym. Sci. Part B Polym. Phys. 2005, 43, 22692279.

(20) Wu, H.-L.; Yang, Y.; Ma, C. M.; Kuan, H. J. Polym. Sci. Part A Polym. Chem. 2005, 43, 6084-6094.

(21) Fukushima, T.; Kosaka, A.; Ishimura, Y.; Yamamoto, T.; Takigawa, T.; Ishii, N.; Aida, T. Science 2003, 300, 2072-2074.

(22) Oh, K.; Lee, J. Y.; Lee, S.-S.; Park, M.; Kim, D.; Kim, H. Compos. Sci. Technol. 2013, 83, 40-46.

(23) Das, A.; Stöckelhuber, K. W.; Jurk, R.; Fritzsche, J.; Klüppel, M.; Heinrich, G. Carbon 2009, 47, 3313-3321.

(24) Subramaniam, K.; Das, A.; Steinhauser, D.; Klüppel, M.; Heinrich, G. Eur. Polym. J. 2011, 47, 2234-2243.

(25) Steinhauser, D.; Subramaniam, K.; Das, A.; Heinrich, G.; Klüppel, M. Express Polym. Lett. 2012, 6, 927-936.

(26) Likozar, B. Sci. Iran. 2010, 17, 35-42.

(27) Subramaniam, K.; Das, A.; Häußler, L.; Harnisch, C.; Stöckelhuber, K. W.; Heinrich, G. Polym. Degrad. Stab. 2012, 97, 776-785.

(28) Subramaniam, K.; Das, A.; Simon, F.; Heinrich, G. Eur. Polym. J. 2013, 49, 345-352.

(29) Subramaniam, K.; Das, A.; Heinrich, G. Compos. Sci. Technol. 2011, 71, 1441-1449.

(30) Daugaard, A. E.; Hassouneh, S. S.; Kostrzewska, M.; Bejenariu, A. G.; Skov, A. L. 2013, 8687, 868729-868729-11.

(31) Daugaard, A. E.; Jankova, K.; Marín, J. M. R.; Bøgelund, J.; Hvilsted, S. Eur. Polym. J. 2012, 48, 743-750.

(32) Madsen, F. B.; Yu, L.; Daugaard, A. E.; Hvilsted, S.; Skov, A. L. RSC Adv. 2015, 5, $10254-$ 10259.

(33) Grady, B. P. Macromol. Rapid Commun. 2010, 31, 247-257. 